\title{
LWDSA: light-weight digital signature algorithm for wireless sensor networks
}

\author{
M LAVANYA*(D) and V NATARAJAN \\ Department of Instrumentation Engineering, Anna University, Chennai 600025, India \\ e-mail: drop2lavi@gmail.com
}

MS received 18 May 2016; revised 31 January 2017; accepted 13 February 2017; published online 14 September 2017

\begin{abstract}
The essential security mechanism in wireless sensor networks (WSNs) is authentication, where nodes can authenticate each other before transmitting a valid data to a sink. There are a number of public key authentication procedures available for WSN in recent years. Due to constraints in WSN environment there is a need for light-weight authentication procedure that consumes less power during computation. This proposed work aims at developing a light-weight authentication protocol using MBLAKE2b with elliptic curve digital signature algorithm (ECDSA). The proposed protocol is also tested using the protocol verification tool Scyther and found to be secure in all claims and roles. This proposed algorithm increases the network life time and reduces the computation time, which is essential for the constrained environment like WSNs.
\end{abstract}

Keywords. Wireless sensor networks; authentication; elliptic curve cryptography; sponge function; hash code; digital signature algorithm.

\section{Introduction}

Wireless sensor network (WSN) can be considered as a special type of ad-hoc network containing a huge number of small resource-constrained devices. WSN is becoming the next generation network with advanced technologies in ubiquitous computing. They have a wide variety of applications in military, habitat monitoring, disaster detection, health care, smart building, smart homes, smart vehicles, etc. The sensors used in such networks are usually battery powered, and hence protocols and frameworks to be used in WSN are designed to use energy as efficiently as possible so that the network lifetime can be increased. Most of the WSN applications do not even consider the security aspects because of the heavy computations involved in security algorithms. However, when the network is monitoring and transmitting confidential information, security becomes an important issue that needs to be addressed. Even if the data transmitted are not confidential, it is essential to ensure the authenticity of the neighbour to whom the data are transmitted and the receiver should be confident that the data are not modified (data integrity). There are many security requirements for a wireless network like confidentiality, data integrity, authentication, non-repudiation and availability. All the requirements are not necessarily satisfied by the network; requirements may vary depending on the application. Some applications emphasize data confidentiality irrespective of authentication, while some

*For correspondence applications lay emphasis on authentication. Hence, the security framework of a network depends on the application and the area where the network is going to be deployed.

This paper is organized as follows. Section 2 introduces the related works in the field of authentication in WSN. Section 3 has the preliminaries; The concepts of elliptic curve, elliptic curve cryptography (ECC) and sponge functions are explained in detail. Section 3 provides details of the MBLAKE2b hash function. Section 4 provides the details of the proposed network model and outlines the assumptions. Section 5 explains the proposed authentication scheme in detail. Section 6 gives the highlights of the proposed work. Section 7 evaluates and discusses the results of the proposed work. Security of the proposed work is analysed in section 8. Section 9 explains the verification of the proposed protocol using the tool Scyther. Section 10 gives a comparison of the proposed protocol against the existing authentication protocols. Section 11 concludes the work.

\section{Related work}

In the present scenario there are many cryptographic algorithms providing the security framework for WSN. Both asymmetric and symmetric ciphers are used in WSN as shown in figure 1 .

TinySec, LEAP and SPINS are based on symmetric key cryptographic techniques and TinyPK, TinyECC, TinyPBC, 


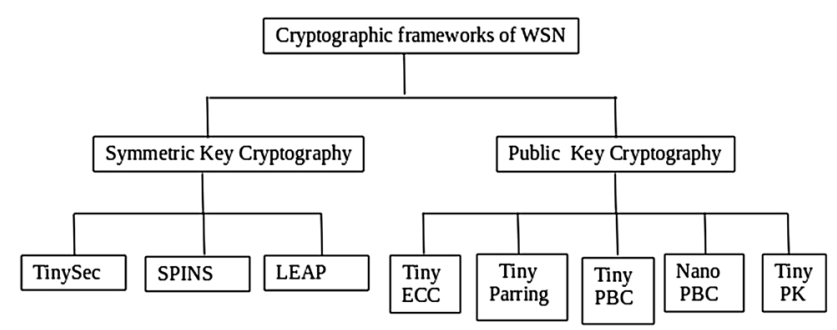

Figure 1. Cryptographic frameworks for WSN.

NanoPBC and Tinypairing are based on public key cryptographic systems. TinySec was the first data-link layer security protocol introduced by Karlof et al. It uses a message authentication code (MAC) for authentication and provides two modes of operation: Tinysec Authenticated encryption and Tinysec Authentication mode. The drawback in Tinysec is the key management, as it uses a single key for generating MAC. Hence, there is a single point of failure. SPINS was proposed by Perrig et al. Secure network encryption protocol (SNEP) is one of the components of SPINS. The two most important security requirements, confidentiality and twoparty authentication, are provided by SNEP. Localized Encryption and Authentication Protocol (LEAP) was proposed by Zhu et al. The principle behind LEAP is that the nodes require different levels of security depending on the message exchanged. Hence, many pre-shared network keys were used based on the assumption that the keys are not disclosed at the initial time $t$. It does not address the packet loss and feedback implosion.

The algorithms like PRESENT, HIGHT and CURUPRIA-2 are proposed for providing security in constrained environment. All these algorithms concentrate on the confidentiality part of security. Another vital security requirement is the authentication. Authentication is usually provided by generating a MAC. MAC is generated using CBC-MAC or uses design techniques like Carter-Wegman design or ALRED construction. One of the MAC algorithms that uses the

Carter-Wegman design technique is GMAC, which uses

GHASH. The algorithms using the ALRED design are SHARK, SQUARE, PELICIAN and MARVIN.

From the conclusion of [1] work, it is possible to use public key cryptography (PKC) in WSN; it is clear that PKC is possible on small devices. PKC in the field of authentication provides simple solutions, strong security resilience, good scalability and immediate node authentication. The TinyPK is based on RSA; TinyECC is based on ECC; TinyPBC, Tinypairing and NanoPBC are based on parring-based cryptography. There are many other recently developed frameworks like Sensec [2], Minisec [3] and Contikisec [4] providing similar security services and aiming at minimizing energy. PKC can be used for broadcast authentication, which is one of the important communication concepts of WSN. An authentication scheme based on certificates was proposed in [5], and public key certificates are issued by a third party called the Certification Authority (CA). This scheme was found to be inefficient due to its communication and computation overheads, because the certificates are transmitted along with the message. One more important issue is the revocation of certificates and the management of the certificate revocation list (CRL). The CRL should be stored in each node, which increases memory usage. The next PKC technique is the ID-based authentication [6], which has attracted the attention of researchers working in the field of authentication. The public keys of the entities are derived from some characteristics of the ID. The private keys are generated by a trusted third party called the Private Key Generator (PKG). The certificates are not transmitted along with the message; therefore, communication overhead is reduced. However, it incurs a very high computation overhead as it uses bilinear paring [7] operations.

EIBAS [8] uses paring optimal ID-based signature scheme. Compared with all other ID-based signature schemes, this scheme uses the shortest broadcast message size. Since it uses bilinear pairings, the computation cost is high. The drawback of ID-based schemes $[9,10]$ is the key escrow problem because the private keys are generated by the third party. A survey of broadcast authentication protocols for WSN is given in [11], where authentication protocols are compared to 13 different properties; one of the properties for comparison is the cryptographic technique used. Most of the hybrid broadcast authentication protocols use ECDSA; hence, the proposed modified version can be used for enhancing the performance of such protocols. Another broadcast authentication procedure using ECDSA is discussed in [12]; this scheme does not require time synchronization and uses HMAC for the hash code generation. Methods of fast authentication are discussed in [13] and [14]; both of these schemes concentrate on the release of intermediate message by the sensor node while verifying the signatures. Using the intermediate messages the other nodes can verify the signature fast. However, the burden here is the release of intermediate message by nodes and the frequency of the release of the messages, which increases the communication overhead. In the recent past, many certificate-less signature schemes were proposed; one such protocol is discussed in [15] for VANETS; it solves the ID-PKC key escrow problem with bilinear maps and bilinear pairings, which is again computationally inefficient. User authentication schemes for WSN deals with a smart-card-based authentication [16]; here, the user can directly authenticate with the node; one-way hash functions and XOR operations are used, which reduces the computation cost. However, later this technique was proven in [17] to be susceptible to impersonation attack, sensor node spoofing and stolen verifier attack and it also fails to ensure forward secrecy. Another key renewal authentication procedure is discussed in [18], where authentication is done during the key establishment phase and when the keys are renewed the nodes are reauthenticated. 
Inspired by the upcoming authentication frameworks for WSN in the literature, this paper proposes a light-weight, compact, fast message authentication algorithm based on ECC and cryptographic hash functions.

\section{Preliminaries}

\subsection{Elliptic curves}

Elliptic curves are algebraic curves defined over a finite field. It finds abundant application in cryptography and integer factorization. The arithmetic operations defined on elliptic curves [19] are point addition, point doubling and point multiplication. Point doubling is adding a point to itself, i.e., $P+P$, and multiplying a scalar with the point $\mathrm{P}$, i.e., say, $m * P$ is called point multiplication.

The elliptic curves are represented in general by the Weierstrass equation. There are different types of curves depending on the $x$ and $y$ values of the general equation (1):

$$
E: y^{2}+a_{1} x y+a^{3} y=x^{3}+a^{2} x_{2}+a^{4} x+a^{6} .
$$

The following curves with a simplified version of Eq. (1) are used for cryptographic purposes:

$$
\begin{gathered}
y^{2}=x^{3}+a x+b, \\
y^{2}+x y=x^{3}+a x+b .
\end{gathered}
$$

The elliptic curves operate over a finite field $F_{q}$; when $q$ is prime the field is $F_{p}$. Otherwise, if $q$ is binary, then $q$ is represented as $q=2^{m}$; in this case the field becomes $F_{2^{m}}$. In both the cases, there are certain domain parameters of the elliptic curve that have to be distributed to all the communicating parties, when the curves are used for cryptographic purpose. There are standard values for the domain parameters specified in SEC2 [20], which the implementers of ECC can use. Elliptic curve domain parameters over $F_{p}$ is given by

$$
T=(p, a, b, G, n, h) .
$$

The details of the elliptic curve domain parameters are given in table 1 .

The major application of elliptic curves is in the area of cryptography. In the cryptographic context the most important parameter to be considered is the keys used. The size of the keys used in ECC is very small, which makes it suitable for resource-constrained environments like WSN. Table 2 gives the NIST-recommended key size comparison for various cryptographic techniques [21].

\subsection{ECDSA}

ECDSA is the digital signature algorithm using elliptic curves [19]. ECDSA uses public key cryptographic
Table 1. The domain parameters of elliptic curve.

\begin{tabular}{lc}
\hline Parameter & Description \\
\hline$p$ & Prime number $p$ of field $F_{p}$ \\
$a, b$ & Elliptic curve parameters \\
$G$ & Generator of the field \\
$n$ & Order of $G$ \\
$h$ & Cofactor $h=\sharp E\left(F_{q}\right) / n$ \\
\hline
\end{tabular}

Table 2. NIST guidelines: key sizes for different cryptographic algorithms [21].

\begin{tabular}{lccccc}
\hline \multirow{2}{*}{$\begin{array}{l}\text { Security } \\
\text { (bits) }\end{array}$} & $\begin{array}{c}\text { Symmetric key } \\
\text { encryption }\end{array}$ & $\begin{array}{c}\text { Hash } \\
\text { algorithm }\end{array}$ & \multicolumn{3}{c}{$\begin{array}{c}\text { Minimum size of } \\
\text { public keys (bits) }\end{array}$} \\
\cline { 3 - 6 } & & & $\begin{array}{c}\text { DSA/ } \\
\text { DH }\end{array}$ & RSA & ECC \\
\hline 80 & - & SHA-1 & 1024 & 1024 & 160 \\
112 & 3DES & - & 2048 & 2048 & 224 \\
128 & AES128 & SHA256 & 3072 & 3072 & 256 \\
192 & AES192 & SHA384 & 7680 & 7680 & 384 \\
256 & AES256 & SHA512 & 15,360 & 15,360 & 512 \\
\hline
\end{tabular}

technique; public and private keys are generated based on the domain parameters of the specific curve selected for operation. These domain parameters are agreed upon by the group of users in the communication range. Various techniques are used for the creation of domain parameters [20].

3.2a ECDSA key pair generation: In PKC, a pair of keys are needed for cryptographic purpose, the public and the private key. In ECDSA, the key generation is based on the domain parameters of the elliptic curve chosen. A random value selected by a node acts as the private key. Let $d$ be the random value such that $d \in(1, n-1)$. The public key $Q$ is computed as $Q=d G$, where $G$ is the generator point on the elliptic curve.

3.2b ECDSA signature generation and verification: The signature generation and verification depends on the agreed domain parameters among the communicating entities. The public and the private keys $Q$ and $d$, respectively, must be generated before generating the signature.

\section{Generation}

1. Compute $r=x \bmod n$ where $x=k * G$ and $1 \leq k \leq n-1$.

2. Find $k^{-1} \bmod n, e=S H A-1(m), s=k^{-1}(e+d r) \bmod$ $n$. Generated signatures are $s$ and $r$, and are sent to the receiver.

\section{Verification}

1. Check $r, s$ in $[1, n-1]$.

2. Compute $e=S H A-1(m), w=s^{-1} \bmod n, u=e w$ $\bmod n$ and $v=r w \bmod n$. 
3. Find $X=u G+v Q$; if $x=0$, reject the signature, otherwise compute $v=x \bmod n$ and verify $v=r$. Signature is accepted only if $v=r$.

This is the ECDSA used in practice. In the proposed work, a modified version of this algorithm is used.

\subsection{Cryptographic hash functions}

Cryptographic hash functions are used in applications that provide authentication and integrity. These hash functions convert arbitrarily long messages to a fixed-size output called the hash code. Merkle-Damgard construction or sponge construction [22] is used for the construction of the hash functions. There are many cryptographic hash functions like SHA-3, MD5, RIPEMD, etc. Of these, the SHA-3 [23] algorithm introduces the usage of sponge-based constructions. SHA-3 uses Keccak [24] family of sponge functions. The strength of hash function is measured by three properties, namely collision resistance, pre-image resistance and second pre-image resistance [25]. In this paper, the sponge-based constructions of cryptographic hash functions are considered.

3.3a Sponge function: Sponge functions are used for generating hash codes of arbitrary length. A sponge function instantiates a sponge construction. This simple iterated construction takes variable length input and uses a permutation function $f$ having fixed length and operating on $b$ bits. The output of the sponge construction is of arbitrary length. The $b$-bit width is called as a state in sponge construction, where $b=r+c, r$ is the bit rate and $c$ is the capacity [26]. The protocols or the procedure used decides the initial value of the state and sometimes takes a value 0 , otherwise any constant value. The input message is preprocessed, where it is split into blocks of $r$ bits each and padding is done if necessary. There are two phases in the construction of the sponge function absorption and squeezing phase. The input is absorbed in the absorption phase. The first $r$ bits of the state and the $r$ bit input are XORed, and then processed by the permutation function $f$. This process is repeated for all the input blocks. Following absorption phase is the squeezing phase, where the first $r$ bits of the state are squeezed out of the output block. The fixed permutation function $f$ is used in the subsequent stages. Depending on the number of bits in output, the number of blocks in the squeezing phase increases. The output is truncated to the required number of $l$ bits. Only the first $r$ bits are directly affected by the input; the last $c$ bits of the state remain unchanged by the input or the output. However, they do not remain as a constant value because of the permutation function $f$. This value of $c$ determines the security level attained by the construction. The sponge construction is shown in figure 2.

Hash function mode of operation of the sponge function is considered in this paper. The output of the sponge

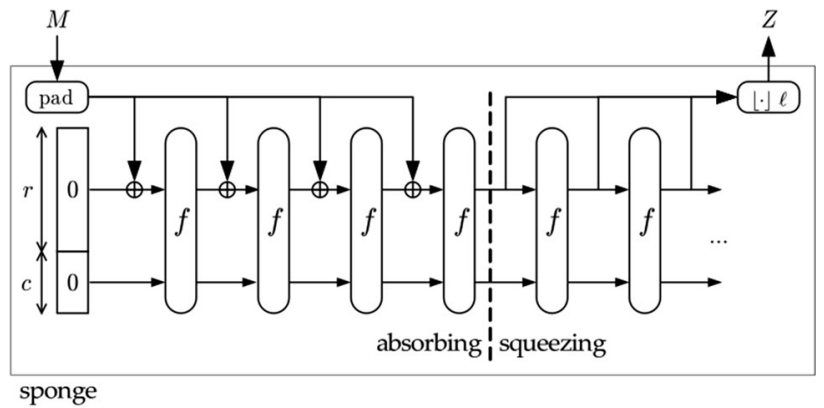

Figure 2. Sponge construction.

function is the message digest, which is truncated to $n$ bits.

\subsection{MBLAKE2b - message digest generation}

In the year 2007, a competition was held for the construction of cryptographic hash function SHA-3. The competition was conducted by NIST. Five third-round candidates were announced in 2010 and the final construction was announced in 2012. One of the third-round candidates is BLAKE [23]. The performances of all the five SHA-3 finalists are analysed. The performances of BLAKE, Keccak and Skein are found to be close [27]. For a hash output up to 256 bits, BLAKE [28] algorithm outperforms all other algorithms. Hence, BLAKE is taken as the basic algorithm and modifications are done to design MBLAKE2b [29]. The compression function of BLAKE is based on wide pipe block cipher, which operates on an inner state represented as a $4 \times 4$ matrix. The state is initialized with IV, salt value and counter in BLAKE. The iteration function of BLAKE is designed such that the second pre-image attack of long messages is avoided. The design of internal blocks as such avoids local collisions. The compression function of a chacha has been modified and used in BLAKE. The compression function of BLAKE is modified in MBLAKE2b. BLAKE uses the initial vectors and salt values for initializing the state. These values are avoided in MBLAKE2 $b$ and hence the memory storage is reduced. The message block is initialized in the state, where it is included in the compression function in BLAKE. Similar to BLAKE there are 12 rounds and each round has $8 G$ functions. These $G$ functions are used in the computation of the diagonal step and the column step, as described in detail in [29]. The diagonal step and the column step can be performed in parallel, thereby reducing the computation time and hardware requirements. The computation time of the generated hash code for fixed size is compared. MBLA$\mathrm{KE} 2 \mathrm{~b}$ is found to be computationally efficient in generating hash code and also in elliptic curve signature generation and verification. Hence, it is more suitable for resourceconstrained environments. 
Table 3. Notations and symbols used.

\begin{tabular}{lc}
\hline Symbol/notation & Description \\
\hline BS & Base station \\
$H\left(I D_{a}\right)$ & Node ID of $A$ \\
$I D_{a}$ & Elliptic curve parameters \\
$P_{U B}^{A}$ & Public key of $A$ \\
$P_{R A}^{A}$ & Private key of $A$ \\
$K_{A B}$ & Session key between $a$ and $b$ \\
$r, s$ & Signature elements \\
$N_{a}$ & Nonce generated by $A$ \\
$k^{\prime}$ & Commitment \\
$\partial$ & Challenge \\
$Z$ & Response \\
\hline
\end{tabular}

Hence, in this modified ECDSA, MBLAKE2b is used for generating the hash code. An authentication framework containing both one-way and mutual authentication is constructed for WSN using digital signatures, without the use of certificates. For a 30-node network the Qos parameters like latency, energy, throughput, etc. are measured.

\section{Network model and assumptions}

In the proposed authentication framework, it is assumed that the network is static and the nodes can communicate with each other and to the base station either directly or by multi-hop communication. The base station is assumed to be resourceful and secure. Sink is the base station. Table 3 gives an explanation for the notations and symbols used in the proposed work.

\section{Proposed authentication scheme}

The six domain parameters of elliptic curve are stored in the sensor node before deployment. The proposed technique has three phases: the registration phase, the authentication phase, where two types of authentication are used - node to node (mutual authentication) and node to base station (one-way authentication) - and the key agreement phase.

The network environment is set by storing the six domain parameters of the elliptic curve in all the nodes. The curve used is Koblitz curve secp160k1. Every node then generates its public and private key.

\subsection{Registration phase}

Node registration with the base station is done here and explained graphically in figure 3 .

Step 1. $A \rightarrow B S: I D_{a} \| P_{U A}^{A}$ : Every node sends its ID and the corresponding public key to the BS.

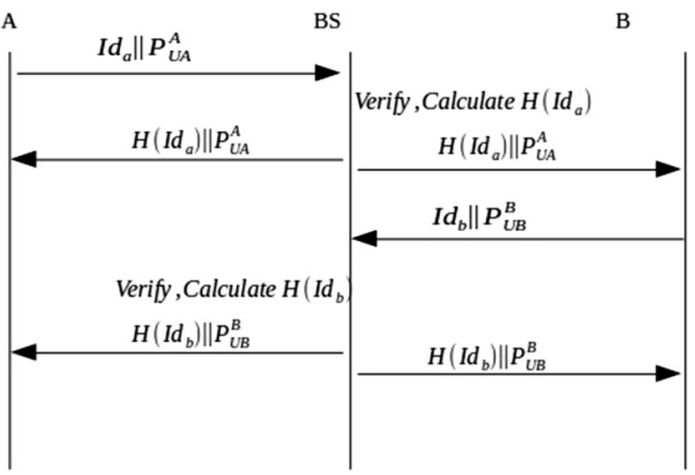

Figure 3. Registration.

Step 2. $\quad B S \rightarrow A, B: H\left(I D_{a}\right) \| P_{U A}^{A}$ : BS verifies with the existing node ID. It generates the hash code for the first node, attaches its public key and broadcasts this data. Similarly, all the nodes send their ID and receive their hash code and its public key.

The nodes receive their neighbours' public key and hash code and store the values in the neighbour table.

\subsection{Authentication phase}

Two different types of authentication are performed mutual authentication between the nodes and one-way authentication for authentication between node and base station as shown in figures 4 and 5 , respectively.

5.2a Node to node authentication: Assume there are two nodes $A$ and $B$ to authenticate each other

Step 1. $\quad A \rightarrow B: I D_{a}\|r\| k^{-1}\left(H\left(I D_{a}+d r \| N_{a}\right)\right.$. Node $A$ generates the signature parameters $r$ and $s$, based on its own private key and transmits its signature parameters along with its ID and a nonce to $B . B$ computes the hash code from the received $I D_{a}$ and compares with the value received from the $\mathrm{BS}$; if the values are equal then it extracts the public key of the corresponding hash code and verifies the signature. If the signature is verified successfully, a secret value $K_{A B}$ is calculated as $K_{A B}=d_{1} P_{U A}^{A}$.

Step 2. $\quad B \rightarrow A: K_{A B} N_{a} \| I d_{b}$. Node $B$ encrypts the nonce using the secret key and transmits it to node $A$ along with the ID of $B$. A calculates the secret value after verifying the nonce and retrieves the public key based on the ID sent by $B$.

Step 3. $A \rightarrow B: K_{A B}(N a-1)$. For the purpose of mutual authentication, node $A$ sends the nonce value encrypted by the secret key to $B$ again. Hence, both are mutually authenticated. 


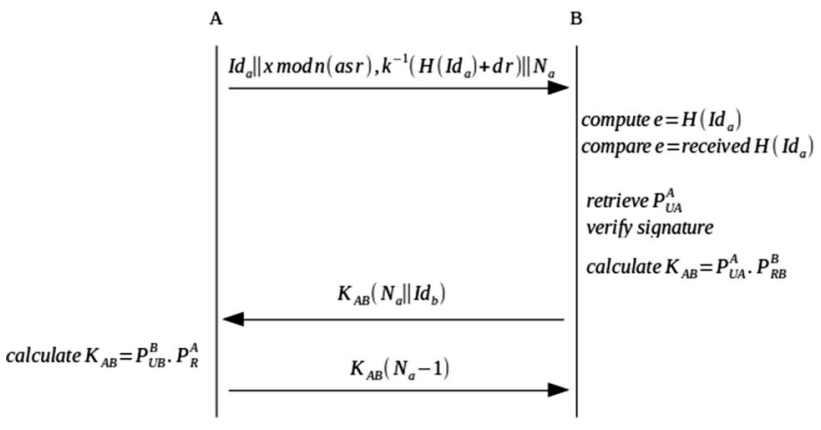

Figure 4. Mutual authentication.

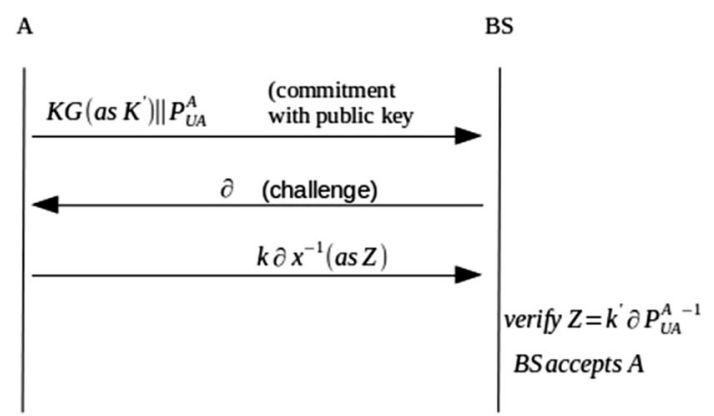

Figure 5. One-way authentication.

5.2b Node to base station authentication: Assume BS wants to authenticate node $A$.

Step 1. $A \rightarrow B S: K^{\prime} \| P_{U A}^{A}$. Node $A$ calculates its commitment as $K^{\prime}=k G$ and transmits this value along with its public key to BS.

Step 2. $B S \rightarrow A: \partial$. BS responds with a random and unique challenge.

Step 3. $A \rightarrow B S: k \partial x^{-1}$. Node $A$ sends a response for the BS to verify $A$. If the received value is equal to $K^{\prime} \partial P_{U A}^{A}{ }^{-1}$ then BS accepts $A$ and broadcasts the hash of ID of $A$ and the public key of $A$. Hence, this is one-way authentication, as it is assumed that the BS is secure and authentic.

\section{Highlights of the proposed protocol}

This section explains the variation of the proposed framework from the existing algorithm, which resulted in the improved performance of the WSN.

\subsection{Choice of the hash function}

The major variation is the replacement of the algorithm used in the generation of hash code (SHA-1) by MBLAKE.
As explained in section 8.2, MBLAKE2b satisfies security criterion of one-way hash function like pre-image resistance, collision resistance and second pre-image resistance. MBLAKE2b follows a sponge-based construction as explained in section 3.3a.

\subsection{Straus-Shamir trick}

Double scalar multiplications are evaluated using this method [30]. For evaluating the value $u P+v Q$ in ECDSA, Shamir's trick has been used. The traditional way of evaluating the expression requires two multiplications and one addition operation. Hence, it costs 21 doublings and $l$ addition ( $l$ is the number of digits in the scalar value). Using Shamir's trick this has been reduced to $l$ doublings and 0.75 addition.

\subsection{Certificate-less authentication}

When PKC is used in providing security the traditional way of distributing the certificates is by using the public key certificates. A CA usually generates the certificates and issues it to the communicating parties securely. In an environment like WSN, a node cannot act as CA, due to a single point of failure, and there may be a frequent change in the $\mathrm{CA}$, which is not an optimal decision, considering security issues. Certificate-less PKC is achieved using a bilinear pairing that is computationally rich and has the key escrow problem. Therefore, this novel authentication technique provides certificate-less authentication with low computation cost.

\subsection{Choice of curve}

Koblitz proposed these types of elliptic curves for cryptographic use. Reference [31] shows that one can compute $k P$ value efficiently for any $k$ value and a point $P$ on the Koblitz curve. Since the scalar multiplications computation is the major part in ECDSA, Koblitz curves are suitable for use in ECDSA.

\section{Evaluation and discussion}

The protocol framework proposed is evaluated and the results are discussed. First, the message digest generated is analysed and then the response of WSNs with the newly generated ECDSA is discussed.

\subsection{Efficiency of the generated message digest}

MBLAKE2b hash function is implemented and tested against its counter SHA-1 algorithm in [29]. It has been 
inferred that the MBLAKE2b outperforms SHA-1 considering the computation time in generation and verification of the digital signatures using ECDSA.

\subsection{Response of WSN for the modified ECDSA}

A 30-node WSN incorporating the modified ECDSA algorithm in simulated in NS-2. The traditional ECDSA algorithm is implemented for the same framework and the performances of the modified and the traditional ECDSA algorithms are compared in this section. The energy consumption in the network for the traditional and modified ECDSA is shown in figure 6 and the latency comparison is shown in figure 7.

The network lifetime and the throughput comparisons are shown in figures 8 and 9 , respectively.

From figures 7 and 10 it is inferred that there is a significant change in the latency and the packet delivery ratio on comparing the traditional ECDSA algorithm with the modified ECDSA algorithm. Energy consumed by the nodes is less in the proposed work. The number of alive nodes is more at any particular instant of time; after simulation time of $80 \mathrm{~s}$, the number of alive nodes is more by 15 percent than by the traditional algorithm. This implies that there is an increase in the network lifetime. The computation time of traditional ECDSA is $19.756 \mathrm{~ms}$ and that of the modified ECDSA is 16.876 ms.

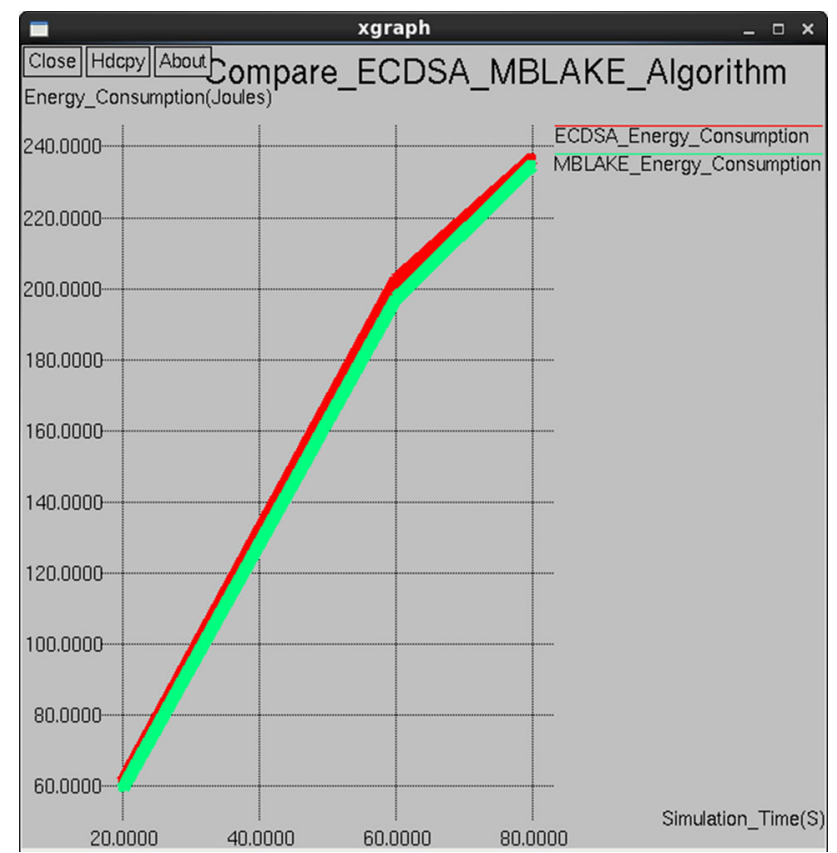

Figure 6. Energy consumption.

\section{Security analysis}

This section focuses on analysing the strength of the proposed protocol based on standard security models and definitions.

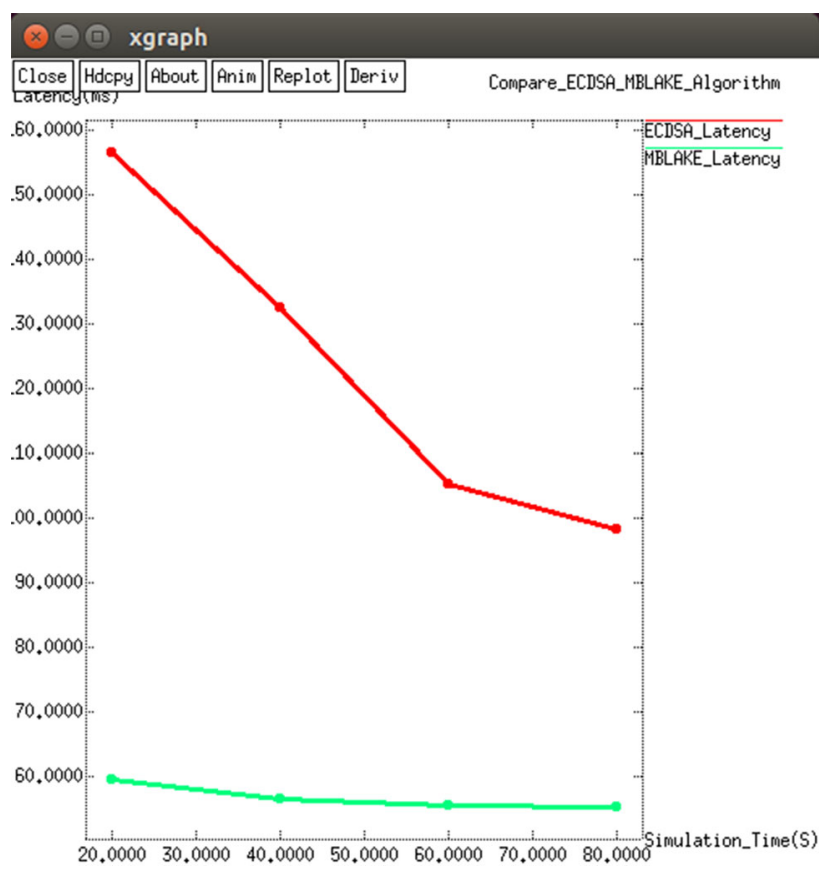

Figure 7. Network latency.

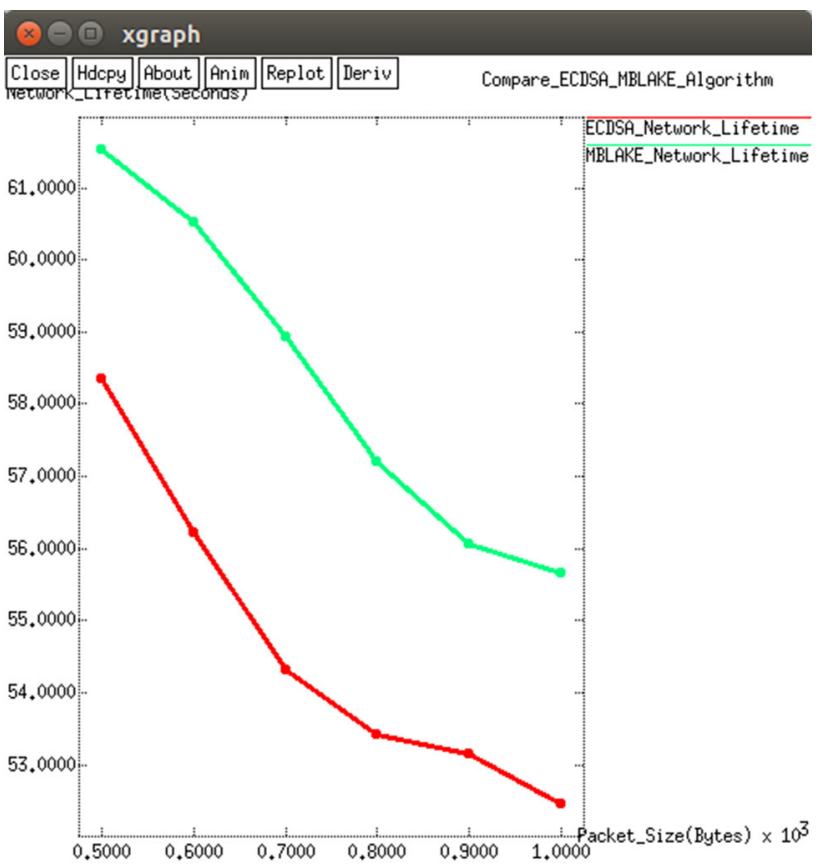

Figure 8. Network lifetime. 


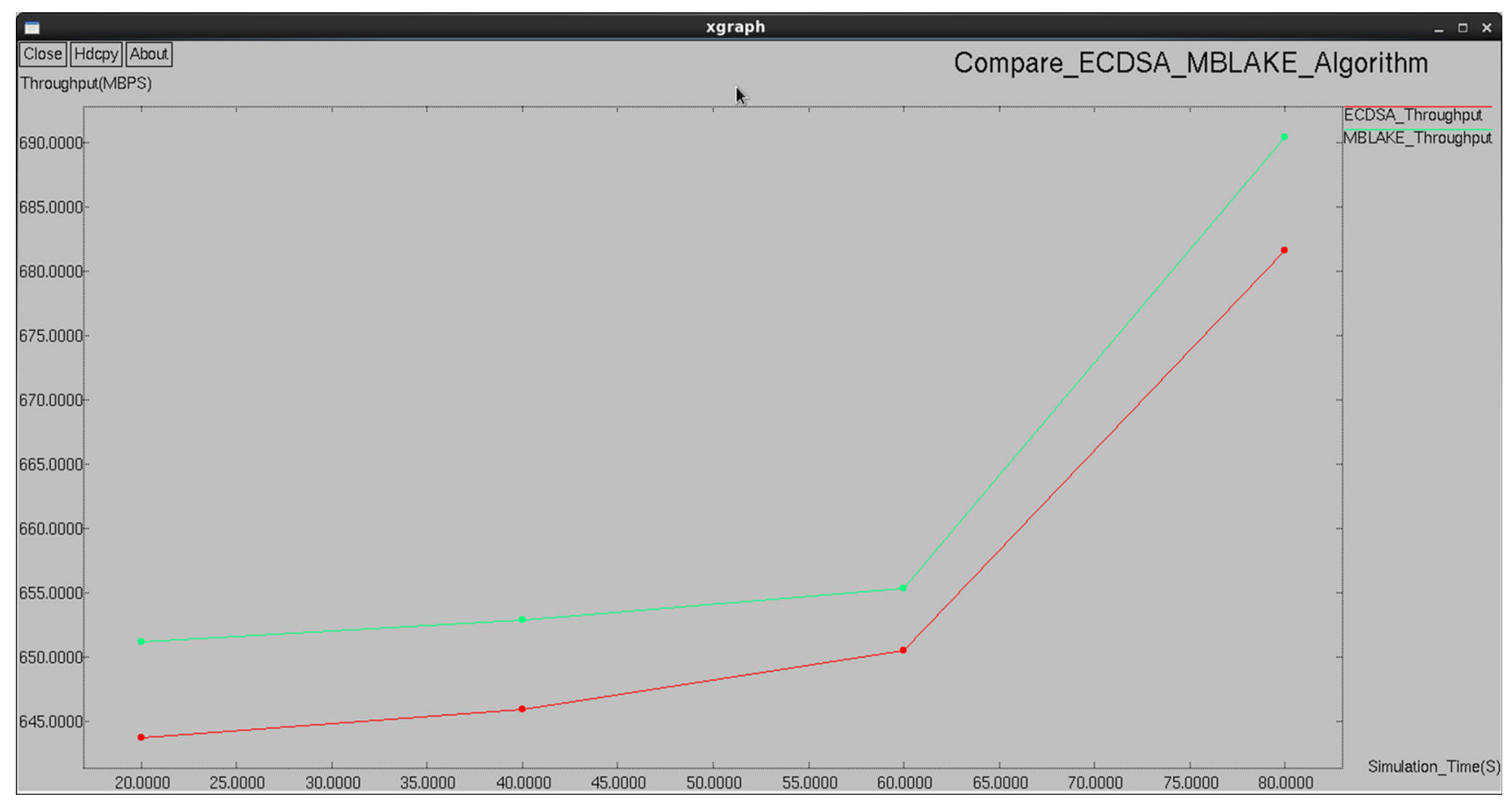

Figure 9. Throughput comparisons.

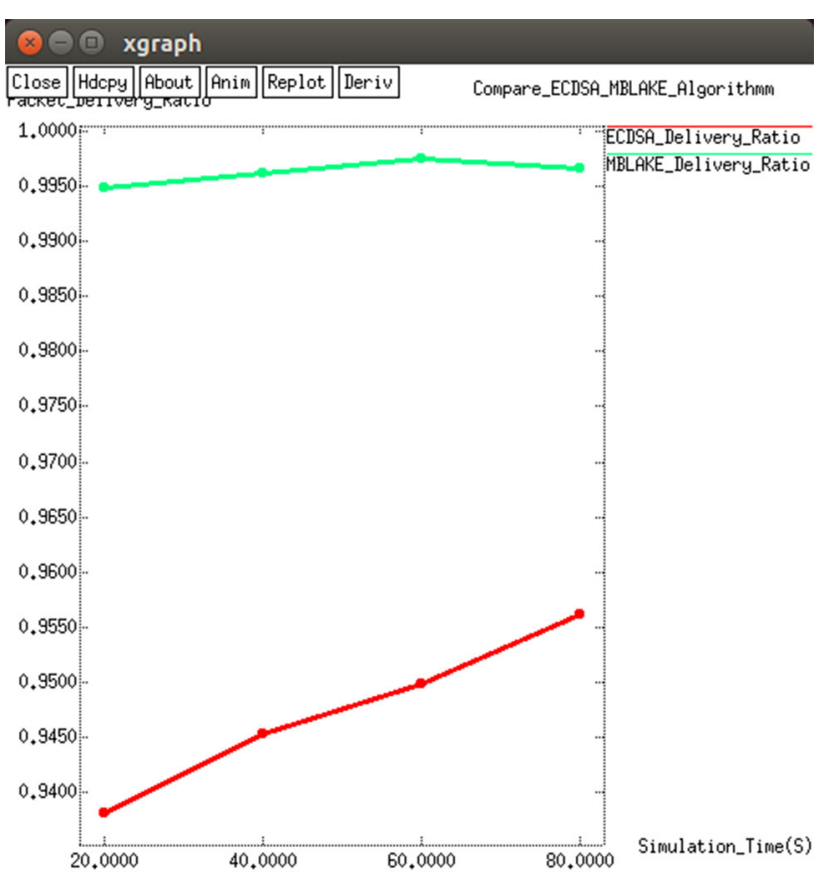

Figure 10. Packet delivery ratio.

\subsection{Security of the proposed framework}

The proposed authentication scheme is based on the challenge-response technique for one-way authentication, where the base station sends a random and unique challenge for each node. Mutual authentication is based on the modified elliptic curve digital signature algorithm. The message digest is constructed using MBLAKE, one-way hash function. The adversary will not be able to forge in both the phases of authentication. Many standard attacks can be restricted as discussed here.

8.1a Man in the middle attack: There are various techniques for overcoming the man in the middle (MITM) attack in literature [32]. Based on the measured RTT and Wi-fi RSS the MITM is detected and located. Machine learning algorithms are used on the RSS dataset for the estimation of the location of the attacker.

In the proposed algorithm during the registration phase, the base station compares the ID of the node and generates its hash value $H(I D)$ and broadcasts the public key and the hash value of the node. Therefore the base station can easily identify the intruder in the middle, whose details are not in the table. MITM is overcome in the registration phase

Next, coming to the mutual authentication phase, neighbouring nodes receive the public key and the corresponding hash value from the base station. Consider that a node (say $A$ ) transmits its ID; signature components $r$ and $s$ are calculated as $x \bmod n$ and $k^{-1}\left(H\left(I D_{a}\right)+d r\right)$, respectively. The signature includes the private key of the sender, which cannot be forged by the intruder because of the strongest discrete logarithm. The hash code included in the signature is generated using one-way cryptographic hash functions, which is as strong as the random oracle model; hence, an intruder cannot forge the signature. Moreover, the receiver verifies the hash value of the received ID and the 
one stored in its database; the verification process starts only if they are equal.

In one-way authentication phase, the BS sends a random and a unique challenge for every node. BS is assumed to be strong and unforgeable; hence, MITM is prevented.

8.1b Mutual authentication: In the node to node communication, node $A$ sends a nonce, which can be a unique value or a time stamp in its first message; after verification, node $B$ retransmits the nonce and its public key encrypted with the secret key in the second message; now the node $A$ verifies the public key in its database, calculates the secret key and transmits the $E_{K_{A B}}\left(N_{a}-1\right)$ to $B$ for authentication. Hence, mutual authentication is achieved.

8.1c Key revocation: The key revocation techniques in WSN are classified as centralized, distributed, decentralized and hybrid schemes [33]. All these methods concentrate on revoking the compromised node in symmetric key cryptography. The key revocation in PKC is equivalent to the certificate revocation. Certificates are revoked when the private key of a node is compromised and the corresponding public key has to be revoked. For this, the CA will issue the CRL, which is stored in the nodes. A revocationfree public key encryption is proposed in [34], which is based on bilinear pairings, which are expensive for the constrained environments. The proposed work in this paper is a certificate-free PKC where there are no CAs and CRLs. The private keys are used in the ECDSA. It is impossible to find the discrete logarithm of a random element with a public parameter in elliptic curves. Hence, the public keys need not be revoked.

8.1d Replay attack: In the proposed work, the base station transmits a unique random challenge for every node; hence, replay is avoided in node to base station communication. In case of mutual authentication the nonce transmitted is a unique value or a time stamp; on receiving the first message of mutual authentication the receiver checks whether it is within the time stamp; thus the transmitted message is not a replay.

8.1e Distributed DoS attack: DDoS attacks are launched by Zombies or Botnet computers. These devices are remotely controlled and widely scattered. They send continuous and simultaneous traffic to the target system. Many DDoS attacks were launched since 1999; the most recent one was in September 2012, which targeted the online banking sites of 9 popular banks of the USA. Powerful DDoS flooding was launched by a hackvist group called Izz Ad-Din al Qassim cyber fighters [35]. Pairwise authentication, where bidirectional links of the nodes are verified before constructing the route, can combat the attack [36]. The requests should be authenticated, refusing to answer even with negative ack.

In the proposed framework, the base station BS is assumed to be secure and resourceful and it cannot be duplicated. Hence, during step 1 of one-way authentication phase, the BS, upon receiving the ID of the nodes, generates $H(I D)$ and verifies with the already generated and stored $H(I D)$ and then broadcasts the message in step 2 . Hence, DDoS can be prevented. When considering the mutual authentication phase, if the received hash value in step 1 of mutual authentication phase is equal to the hash value sent by the base station, then signature verification starts. Hence, DDoS is prevented.

\subsection{MBLAKE2b security}

The three main security properties required by cryptographic hash functions are pre-image resistance, second pre-image resistance and collision resistance. With these three properties let $\complement_{F}{ }^{\text {prop }}$, where prop $\in$ (pre, secpre, collres), represent the maximum gain of an adversary to break the compression function $F$ under prop. Complexity of an attack is measured by the number of queries $q$ to the primitive made by the adversary. In addition to all these properties, indifferentiality property is also taken into consideration $[37,38]$, to ensure there are no structural defects in $F$. The general design strategy for any hash function is that it should have prefix- and suffix-free padding and chop function that drops some bits of the output for generating the hash code. BLAKE follows HAIFA [39] construction, which uses salts and constant values. MBLAKE2b follows a structure similar to that of HAIFA, omitting the counter value and the salt value. Similar to HAIFA, MBLAKE2b preserves the collision resistance and is resistant against the second pre-image attack. MBLAKE2b follows a suffix- and prefix-free padding rule that eliminates the length extension attack. The design preserves collision resistance where $\complement_{H}^{c o l}=\Theta\left(q^{2} / 2^{n}\right)$, and is secure against the second pre-image attack $\complement_{H}^{p r e}=\Theta\left(q / 2^{n}\right)$, where $n$ is the string length of output bits. MBLAKE2b is indifferentiable against the random oracle.

Considering the security of ECDSA, the attacks on ECDSA mainly concentrate on the hash function and the ECDLP. The signature can be compromised if the hash function is not pre-image resistant and collision resistant. Signature repudiation is possible if it is not collision resistant and the signatures can be forged if it is not collision resistant. The solution is to use a variable length hash function. Hence, MBLAKE2b will be a better choice compared with the other cryptographic hash functions.

\section{Security verification of the protocol}

The formal security analysis of the proposed authentication framework is done using the security protocol verification tool Scyther [40]. Security properties like secrecy and authentication are characterized in terms of aliveness; synchronization and message agreement are 


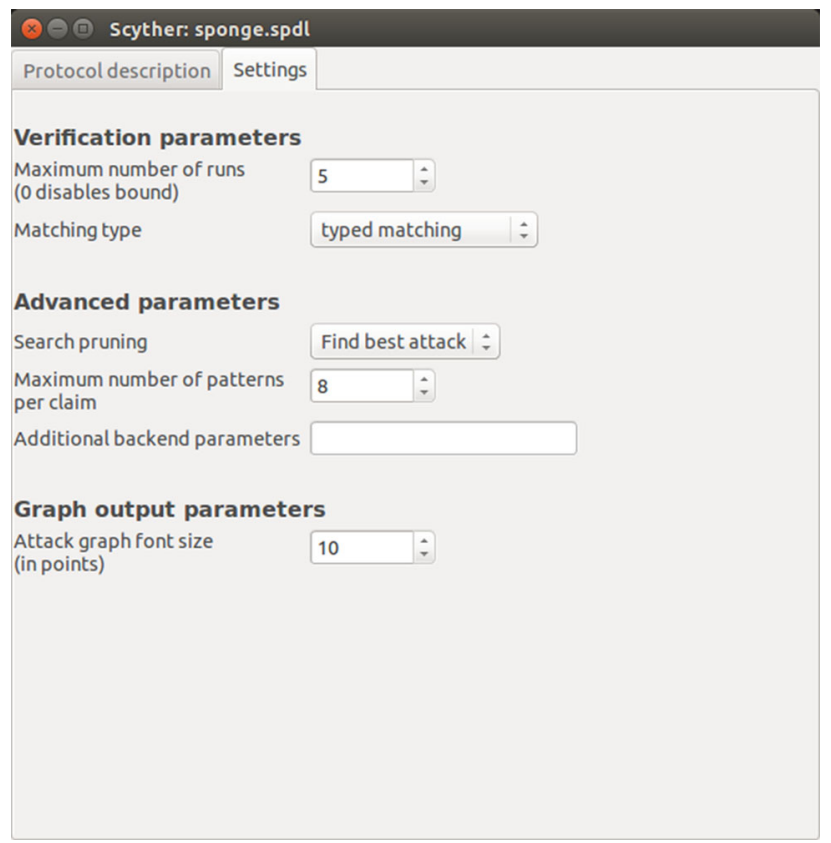

Figure 11. Settings of the tool.

verified using trace patterns. The trace pattern helps in capturing the class of all attack traces for a given protocol and security property. If there are any attack patterns in any specific traces, then it is concluded that the security property is false.

Scyther uses the security protocol verification language (SPDL) for implementing the authentication framework. Here, three role terms are considered, the base station (BS) and two communicating parties A and B. The protocol is analysed for five runs. The settings for the tool is shown in figure 11. Authentication properties like aliveness, non-injective agreement (Niagree) and non-injective synchronization (Nisynch) are analysed. All these properties explain the availability of both partners of communication during every run of the protocol. Aliveness guarantees the initiator the aliveness of another agent who is running the same protocol. Niagree explains about the mutual authentication property, where for an initiator, the protocol steps guarantee that there is a responder for each and every run of the protocol, whereas Nisynch gives an additional confirmation that both send and receive actions are performed in expected order. Both the phases of authentication mechanism in the proposed work are tested with the tool. Figures 12 and 13 give the results of one-way authentication between the BS and the node.

Figure 12 implies that there are no issues in the claims specified in the protocol, but from figure 13 it is understood that the alive and weak agree properties for the BS are not satisfied. The trace patterns for the two properties are shown in figures 14 and 15, which implies that there is an intruder (C) who claims to be the BS. However, the most important assumption of this network is that the BS is

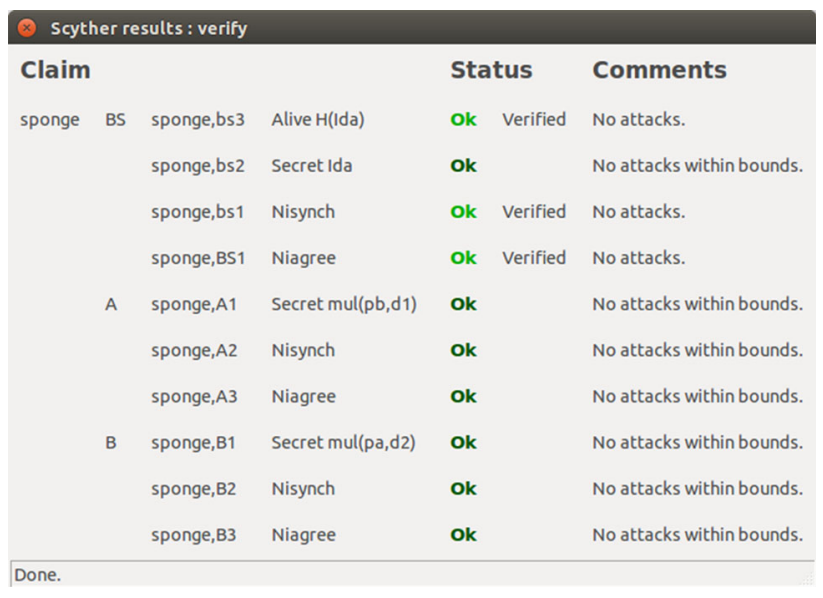

Figure 12. One-way authentication user claim verification.

\begin{tabular}{|c|c|c|c|c|c|c|c|}
\hline \multirow{19}{*}{$\begin{array}{l}\text { Claim } \\
\text { sponge }\end{array}$} & \multirow{7}{*}{ BS } & & & \multicolumn{2}{|c|}{ Status } & Comments & Patterns \\
\hline & & sponge, BS1 & Secret Idb & ok & & No attacks within bounds. & \\
\hline & & sponge, BS2 & Secret Ida & ok & & No attacks within bounds. & \\
\hline & & sponge, BS3 & Alive & Fail & Falsified & Exactly 1 attack. & 1 attack \\
\hline & & sponge,BS4 & Weakagree & Fail & Falsified & Exactly 1 attack. & 1 attack \\
\hline & & sponge, BSS & Niagree & ok & Verified & No attacks. & \\
\hline & & sponge, BS6 & Nisynch & ok & Verified & No attacks. & \\
\hline & A & sponge, $A 2$ & Secret $\mathrm{Na}$ & ok & & No attacks within bounds. & \\
\hline & & sponge, $\mathrm{A} 3$ & Secret Idb & $\mathbf{o k}$ & & No attacks within bounds. & \\
\hline & & sponge, $\mathrm{A} 4$ & Secret Na & ok & & No attacks within bounds. & \\
\hline & & sponge, $A 5$ & Secret Ida & ok & & No attacks within bounds. & \\
\hline & & sponge,As & Secret d1 & ok & & No attacks within bounds. & \\
\hline & & sponge, $\mathrm{A} 7$ & Secret k1 & ok & & No attacks within bounds. & \\
\hline & & sponge, $A 8$ & Secret E & ok & & No attacks within bounds. & \\
\hline & & sponge,A9 & Secret digB & ok & & No attacks within bounds. & \\
\hline & & sponge, $\mathrm{A} 10$ & Alive & $\mathbf{o k}$ & & No attacks within bounds. & \\
\hline & & sponge, A11 & Weakagree & ok & & No attacks within bounds. & \\
\hline & & sponge, $A 12$ & Niagree & ok & & No attacks within bounds. & \\
\hline & & SDonoe.A13 & Nisvnch & ok & & No attacks within bounds. & \\
\hline
\end{tabular}

Figure 13. One-way authentication automatic claim verification.

assumed to be secure and resourceful. Hence, there cannot be an intruder $\mathrm{C}$ who can take the position of the BS. Oneway authentication is done during the registration phase of the node with the BS.

Figures 16 and 17 specify the results of mutual authentication between two nodes. It is inferred from the figures that both user claim and automatic claim verifications indicate no trace of attacks in the protocol.

\section{Performance comparison}

In this section, performance of the proposed authentication framework is compared with the existing recent authentication mechanism in $[8,41,42]$. The comparisons are 


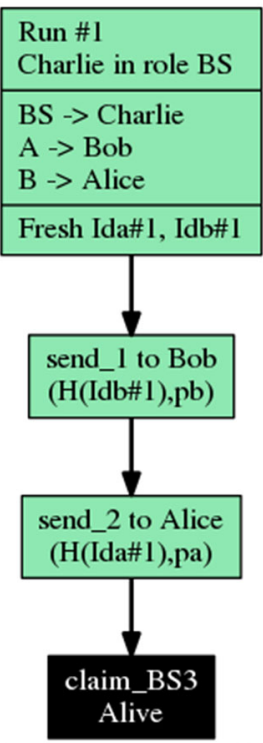

[Id 1] Protocol sponge, role BS, claim type Alive

Figure 14. Trace pattern for alive.

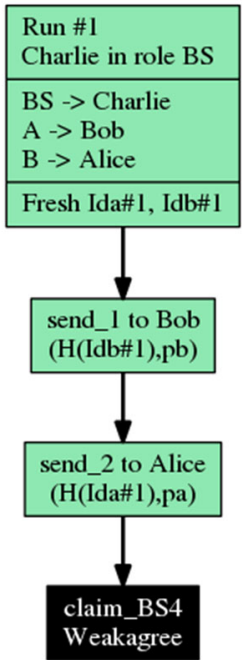

[Id 2] Protocol sponge, role BS, claim type Weakagree

Figure 15. Trace for weak agree.

followed by the explanation of each authentication framework in detail.

\subsection{Shim et al}

EBIAS [8] provides broadcast authentication using the IDbased cryptography, which uses bilinear mappings over elliptic curves called pairings (IBS scheme) with message recovery.

- System initialization: System parameters are generated before deploying the network.

\begin{tabular}{|llllll|}
\hline \multicolumn{2}{|l|}{ Scyther results: verify } & & \\
\hline \multicolumn{2}{|l}{ Claim } & & & Status & Comments \\
sponge & A & sponge,A1 & Secret mul(pb,d1) & Ok & No attacks within bounds. \\
& sponge,bs1 & Nisynch & Ok & No attacks within bounds. \\
& sponge,A2 & Niagree & Ok & No attacks within bounds. \\
& B & sponge,B1 & Secret mul(pa,d2) & Ok & No attacks within bounds. \\
& sponge,B2 & Nisynch & Ok & No attacks within bounds. \\
& sponge,B3 & Niagree & Ok & No attacks within bounds. \\
\hline Done. & & & & \\
\hline
\end{tabular}

Figure 16. Mutual authentication user claim verification.

\begin{tabular}{|c|c|c|c|c|}
\hline \multirow{19}{*}{$\begin{array}{l}\text { Claim } \\
\text { sponge }\end{array}$} & & & Status & Comments \\
\hline & sponge,A2 & Secret Na & ok & No attacks within bounds. \\
\hline & sponge,A3 & Secret Idb & ok & No attacks within bounds. \\
\hline & sponge,A4 & Secret Na & ok & No attacks within bounds. \\
\hline & sponge,A5 & Secret Ida & ok & No attacks within bounds. \\
\hline & sponge,A6 & Secret d1 & ok & No attacks within bounds. \\
\hline & sponge,A7 & Secret k1 & ok & No attacks within bounds. \\
\hline & sponge,A8 & Secret E & ok & No attacks within bounds. \\
\hline & sponge,A9 & Alive & $\mathbf{o k}$ & No attacks within bounds. \\
\hline & sponge, $\mathrm{A} 10$ & Weakagree & ok & No attacks within bounds. \\
\hline & sponge,A11 & Niagree & $\mathbf{o k}$ & No attacks within bounds. \\
\hline & sponge, $\mathbf{A} 12$ & Nisynch & ok & No attacks within bounds. \\
\hline & sponge, $\mathbf{B 2}$ & Secret_Hidden_2 & ok & No attacks within bounds. \\
\hline & sponge, B3 & Secret_Hidden_1 & ok & No attacks within bounds. \\
\hline & sponge,B4 & Secret Idb & ok & No attacks within bounds. \\
\hline & sponge, B5 & Secret pb & ok & No attacks within bounds. \\
\hline & sponge,B6 & Secret d 2 & $\mathbf{o k}$ & No attacks within bounds. \\
\hline & sponge, B7 & Secret Na & ok & No attacks within bounds. \\
\hline & sponqe, B8 & Secret Ida & ok & No attacks within bounds. \\
\hline
\end{tabular}

Figure 17. Mutual authentication automatic claim verification.

1. With the private value $k \in Z^{+}$, prime $q$, groups $G_{1}, G_{2} \quad$ a generator $\mathrm{P} \in G_{1} \quad$ and pairing $e: G_{1} X G_{1} \rightarrow G_{2}$

2. Choose random $s \in_{R} Z^{*}{ }_{q}$ and set $P_{p u b}=s P$. Compute $e(P, P)^{-1}$. Now choose cryptographic hash functions

$$
\begin{aligned}
& H: 0,1^{*} \rightarrow Z_{q} *, H_{1}: 0,1^{*} \rightarrow 0,1^{l_{1}+l_{2}}, F_{1}: 0,1_{1}^{l} \\
& \quad \rightarrow 0,1_{2}^{l}, F_{2}: 0,1_{2}^{l} \rightarrow 0,0_{1}^{l}
\end{aligned}
$$

. The parameters are pre-loaded in the sensor node.

- Private key extraction: The private keys are generated by the PKG; in this scheme, sink node acts as the PKG. 
The private key is generated as $P K_{i}=\operatorname{frac} 1 H(i d)+s P$.

- Signature generation: Let $M$ be a message to be signed; private keys are used in generating the signatures. The current time stamp, say $t t_{i}$, is picked. A random value $r_{1} \in_{R}$ $Z^{*} q$ is chosen and $\mu^{r_{1}}$ and $\alpha=H_{1}\left(I D_{i}, t t_{i}, \mu^{r_{1}}\right) \in 0,1^{l_{1}+l_{2}}$ are computed. Compute $\beta=F_{1}(M) \|\left(F_{2}\left(F_{1}(M)\right) \oplus\right.$ $M), r_{2}=[\alpha \oplus \beta]_{10}$ and $U=\left(r_{1}, r_{2}\right) P K_{i}$. The signature is $\sigma_{i}=\left(r_{2}, U\right)$. The signature component, node ID and the time stamps are broadcasted.

- Authentication: On receiving the broadcast message the time stamp is checked to be valid and the signature verification is done as follows.

Compute $\quad \widetilde{\alpha}=H_{1}\left(I D_{1}, t t_{i}, e\left(U, H\left(I D_{i}\right) P+P_{P u b}, \mu^{r_{2}}\right.\right.$ and $\widetilde{\beta}=\left[r_{2}\right]_{2} \oplus \widetilde{\alpha}$. The signature is verified if $l_{2} \mid \widetilde{\beta}=F_{1}(\widetilde{M})$.

\subsection{Ma et al}

In TinyZKP, [41] the authentication is based on the assumption that it is infeasible to factor a large integer in

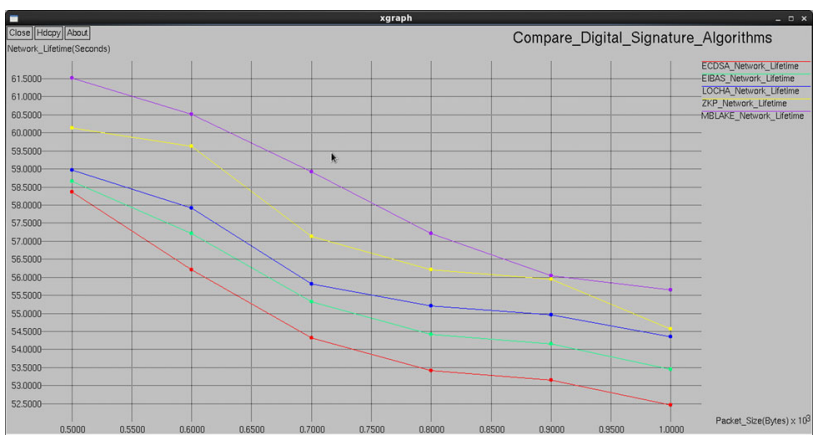

Figure 18. Comparison of network lifetime. polynomial time. The secret keys are generated by a service provider.

Generation of secret keys: There are $n$ pairs of keys between sensor nodes, $\left\{\left[S_{m, 1}, S_{m, 2}, \ldots S_{m, n}\right],\left[V_{m, 1}, V_{m, 2}\right.\right.$.. $\left.\left.V_{m, n}\right]\right\}$. The keys are generated as follows.

An integer $S_{m, 1}$ is selected randomly such that $1 \leq S_{m, 1} \leq N-1, N=p * q$.

$S_{m, j}=S_{m, 1}-j+1(2 \leq j \leq n)$.

Public keys are computed as follows:

$V_{m, j}=\left(\frac{1}{\bmod N}(1 \leq j \leq n)\right.$.

Authentication: The node proves its identity to the BS using the zero knowledge proof algorithm. The BS acts as an authentication centre. First, the BS generates a random unique challenge, computes ECDSA algorithm on the challenge and transmits this signature, random challenges its own ID and a time stamp encrypted with the session key, i.e., $\quad E_{k_{m s}}\left(I D_{b s} \|\right.$ timestamp $\|$ challenge $\mid$ signature $) . \quad$ On receiving this message from the $\mathrm{BS}$ the sensor node decrypts, verifies signature and generates two components $X_{m}$ and $Y_{m}$.

$X_{m}=r^{2} \bmod N, Y_{m}=r \prod_{j=1}^{n} S_{m, j}^{e j}(\bmod N)(1 \leq j \leq n)$.

A message containing the hash value of $X_{m}$, ID of the node, time stamp and $Y_{m}$ is encrypted and sent to the BS. The $\mathrm{BS}$, on receiving the message, computes the time stamp difference and computes the new $X_{m}^{\prime}$ as $X_{m}^{\prime}=Y_{m}^{2} \prod_{j=1}^{n} V_{m, j}^{e j}(\bmod \quad N)(1 \leq j \leq n), \quad$ and checks if received $X_{m}$ equals $X_{m}^{\prime}$. If the values are equal, a new session key is generated and transmitted to the sensor nodes, which in turn reply for the data encrypted with the new session key.

\subsection{Amrita Roy Chowdhury et al}

LOCHA [42] is a cryptographic hash function, which can be used in signature generation and verification. The

Table 4. Comparison of the features of modified ECDSA with the existing recent authentication techniques in WSN.

\begin{tabular}{|c|c|c|c|c|c|}
\hline Feature & Shim et al & Ma et al & $\begin{array}{l}\text { Chowdhury } \\
\text { et al }\end{array}$ & $\begin{array}{l}\text { Traditional } \\
\text { ECDSA }\end{array}$ & Modified ECDSA \\
\hline Key generation & $\begin{array}{r}\text { Private key } \\
\text { generator }\end{array}$ & $\begin{array}{c}\text { Secret keys and public keys } \\
\text { are generated by service } \\
\text { providers }\end{array}$ & Node & Node & Node \\
\hline $\begin{array}{l}\text { Cryptographic } \\
\text { technique } \\
\text { used }\end{array}$ & $\begin{array}{c}\text { Bilinear pairings } \\
\text { over elliptic } \\
\text { curves }\end{array}$ & $\begin{array}{l}\text { Zero knowledge proof } \\
(\mathrm{ZKP})\end{array}$ & $\begin{array}{l}\text { Elliptic curve } \\
\text { cryptography }\end{array}$ & $\begin{array}{l}\text { Elliptic curve } \\
\text { cryptography }\end{array}$ & $\begin{array}{l}\text { Cryptographic hash } \\
\text { functions and elliptic } \\
\text { curve cryptography }\end{array}$ \\
\hline Hash algorithm & & SHA-1 & LOCHA & SHA-1 & MBLAKE \\
\hline Authentication & $\begin{array}{l}\text { Broadcast } \\
\text { authentication }\end{array}$ & User authentication & $\begin{array}{l}\text { One-way } \\
\text { authentication }\end{array}$ & $\begin{array}{c}\text { Mutual } \\
\text { authentication }\end{array}$ & $\begin{array}{c}\text { Mutual and one-way } \\
\text { authentication }\end{array}$ \\
\hline $\begin{array}{l}\text { Authentication } \\
\text { technique } \\
\text { used }\end{array}$ & $\begin{array}{l}\text { Pairing optimal ID- } \\
\text { based signature } \\
\text { scheme }\end{array}$ & $\begin{array}{c}\text { Challenge response } \\
\text { authentication }\end{array}$ & ECDSA & ECDSA & $\begin{array}{c}\text { Modified ECDSA and } \\
\text { Zigma protocol }\end{array}$ \\
\hline $\begin{array}{c}\text { Computation } \\
\text { overhead }\end{array}$ & $\begin{array}{l}\text { Very high due to } \\
\text { bilinear pairings }\end{array}$ & Less & Less & Less & $\begin{array}{l}\text { Reduced by using } \\
\text { MBLAKE } 2 b\end{array}$ \\
\hline
\end{tabular}




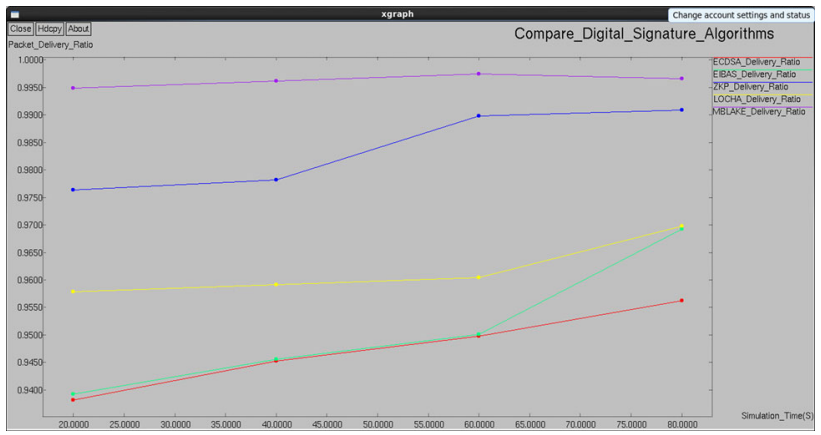

Figure 19. Comparison of packet delivery ratio.

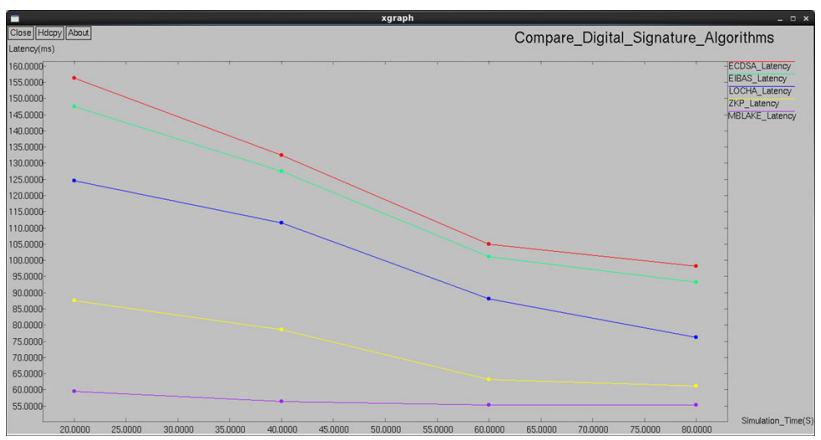

Figure 20. Comparison of network latency.

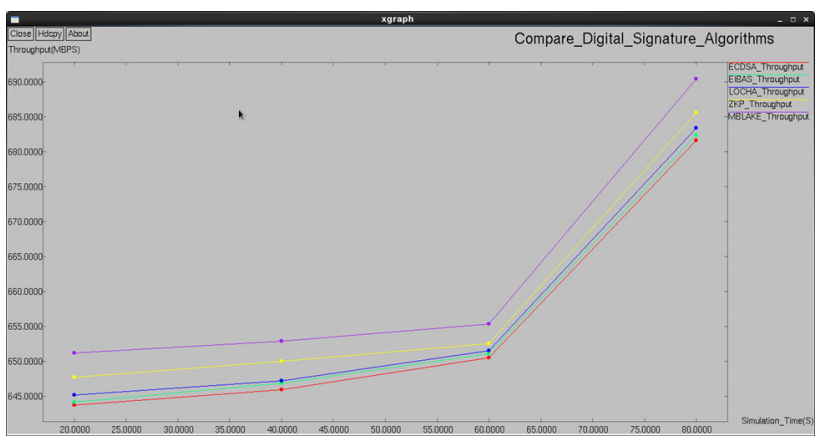

Figure 21. Comparison of throughput.

operations used in the generation of hash code are SWAP and MOD; two substitution boxes are used, input message is preprocessed and divided into blocks of 512 bits, padding is done and three-level swapping is done. This hash function has been implemented and applied in the generation and verification of signatures using ECDSA. The performance of this LOCHA-based signatures is compared to that of the proposed framework.

The QoS parameters of WSN like packet delivery ratio, throughput, latency, network lifetime and energy consumption are measured for the afore-mentioned authentication schemes and values are plotted. Figures 18 and 19

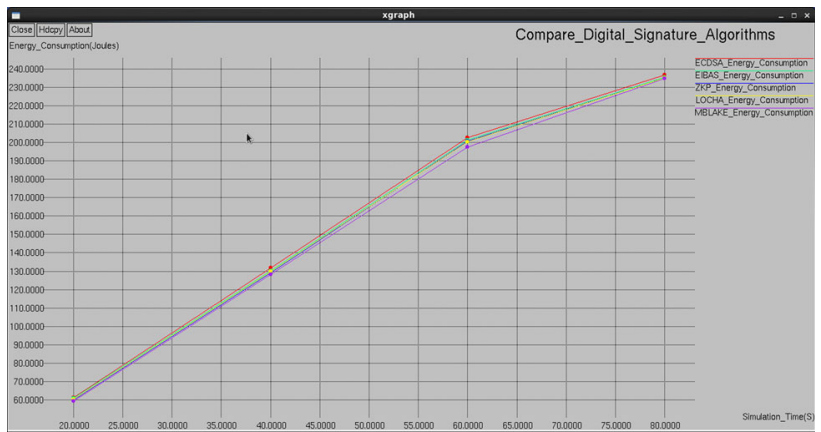

Figure 22. Comparison of energy consumption.

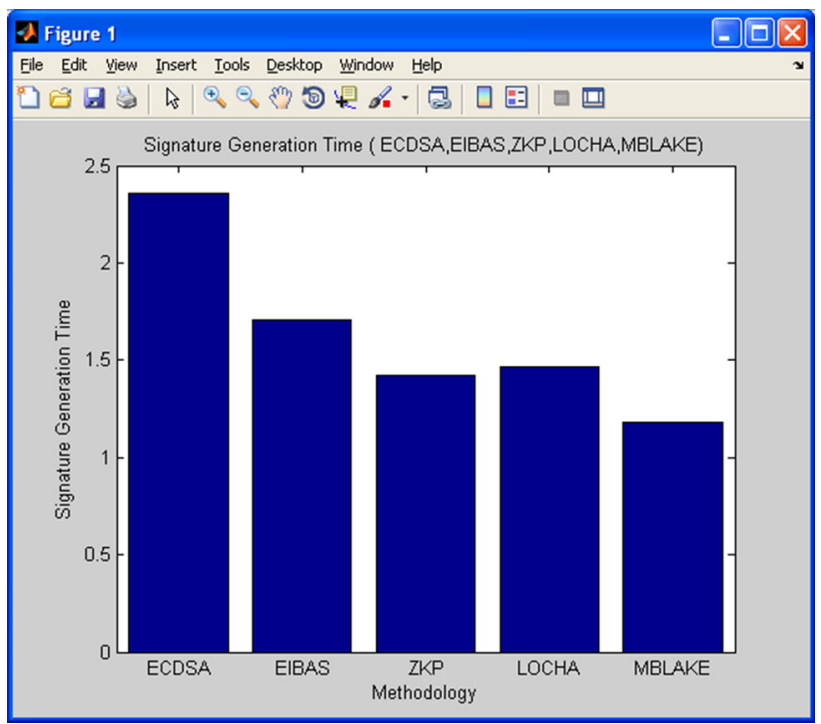

Figure 23. Computation for signature generation.

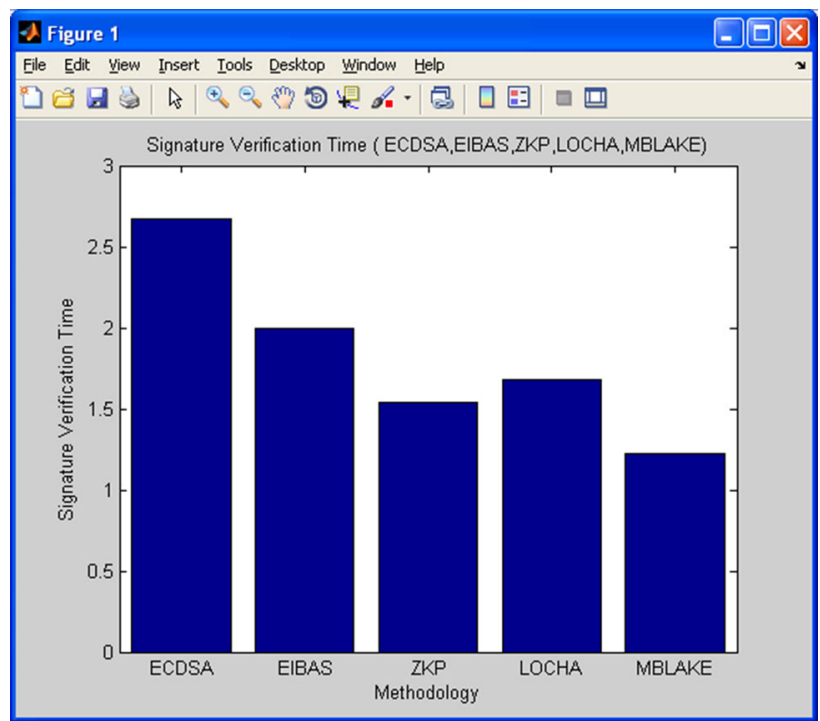

Figure 24. Computation time for signature verification. 
show the network lifetime and the packet delivery ratio. The values of latency and throughput are shown in figures 20 and 21, respectively, and figure 22 shows the energy consumed by the nodes in the network. From these comparisons it is inferred that the proposed authentication framework gives a better performance compared with the existing authentication frameworks. The signature generation and verification time are compared in figures 23 and 24 , respectively. Table 4 gives a comparison of the features of the afore-mentioned authentication procedures of WSN.

\section{Conclusion}

A modified ECDSA incorporating a sponge based hash function for WSN is presented in this paper. From the results obtained through simulation, it is found that the computation time for generating the hash code and the computation time for generating the entire signature and verifying the signature are less compared with the traditional digital signature algorithm. The performance parameters of WSN like energy consumption, throughput, latency and packet delivery ratio are analysed; a better result is found from the proposed algorithm compared with the traditional authentication process.

\section{References}

[1] Wander A, Gura N, Eberle H, Gupta V and Shantz S 2005 Energy analysis of public key cryptography on small wireless devices. In: Proceedings of IEEE PerCom, pp. 324-328

[2] Li T, Wu H, Wang X and Bao F 2005 SenSec design. Technical Report. InfoComm Security Department, USA

[3] Luk M, Mezzour G, Perrig A and Gligor V 2007 MiniSec: a secure sensor network communication architecture. In: IPSN07: Proceedings of the 6th International Conference on Information Processing in Sensor Networks , ACM, New York, NY, USA, pp. 479-488, doi:10.1145/1236360.1236421

[4] Casado L and Tsigas P 2009 Contikisec: a secure network layer for wireless sensor networks under the contiki operating system. In: Proceedings of the Identity and Privacy in the Internet Age 14th Nordic Conference on Secure IT Systems (NordSection 2009), Lecture Notes in Computer Science, vol. 5838, Springer, Berlin/Heidelberg, pp. 133-147, doi:10. 1007/978-3-642-04766-4

[5] Ren K, Zeng K, Lou W and Moran P 2007 On broadcast authentication in wireless sensor networks. IEEE Trans. Wireless Commun. 6(11): 4136-4144

[6] Cao X, Kou W, Dang L and Zhao B 2008 IMBAS: identitybased multi-user broadcast authentication in wireless sensor networks. Comput. Commun. 31: 659-667

[7] Yasmin R, Ritter E and Wang G 2010 An authentication framework for Wireless Sensor Networks using identitybased signatures. In: Proceedings of the 10th IEEE International Conference on Computer and Information Technology (CIT 2010), pp. 882-889. Piscataway, NewJersey, USA: IEEE
[8] Shim K A, Lee Y R and Park C M 2013 EIBAS : an efficient identity-based broadcast authentication scheme in wireless sensor networks. Ad Hoc Netw. 11(1): 182-189

[9] Fan X and Gong G 2012 Accelerating signature-based broadcast authentication for wireless sensor networks. Ad Hoc Netw. 10(4): 723-736

[10] Benzaid C, Lounis K, Al-Nemrat A, Badache N and Alazab M 2016 Fast authentication in wireless sensor networks. Future Gener. Comput. Syst. 55: 362-375

[11] Horng S J, Tzeng S F, Huang P H, Wang X, Li T and Khan M K 2015 An efficient certificateless aggregate signature with conditional privacy-preserving for vehicular sensor networks. Elsevier Inf. Sci. 317: 48-66

[12] Turkanovic B B and Holbl M 2014 A novel user authentication and key agreement scheme for heterogeneous ad hoc wireless sensor networks, based on the Internet of Things notion. Ad Hoc Netw. 20: 96-112

[13] Chang C C and Le H D 2016 A provably secure, efficient, and flexible authentication scheme for ad hoc wireless sensor networks. IEEE Trans. Wireless Commun. 15(1): 357-366

[14] Sung S and Ryou J 2014 ID-based sensor node authentication for multi-layer sensor networks. J. Commun. Netw. 16(4): 363-370

[15] Li F, Zhong D and Takagi T 2012 Practical identity-based signature for wireless sensor networks. IEEE Wireless Commun. Lett. 1(6): 637-640

[16] Liu Y, Li J and Guizani M 2012 PKC based broadcast authentication using signature amortization for WSNs. IEEE Trans. Wireless Commun. 11(6): 2106-2115

[17] Rasheed A and Rabi Mahapatra N 2012 The three-tier security scheme in wireless sensor networks with mobile sinks. IEEE Trans. Parall. Distrib. Syst. 23(5): 958-965

[18] Hankerson D, Menezes A and Vanstone S 2004 Guide to elliptic curve cryptography. New York, NY, USA: Springer-Verlag

[19] Standards for Efficient Cryptography Group (SECG) 2000 Recommended elliptic curve domain parameters. SEC 2

[20] Damgrd I 1989 A design principle for hash functions. In: Brassard G (Ed.) Advances in Cryptology - CRYPTO 89, Proceedings of the 9th Annual International Cryptology Conference, Santa Barbara, California, USA, August 20-24, Lecture Notes in Computer Science, vol. 435, pp. 416-427

[21] Bertoni G, Daemen J, Peeters M and Assche G V 2011 The Keccak reference. Submission to NIST-Round 3

[22] Naor M and Yung M 1989 Universal one-way hash functions and their cryptographic applications. In: Proceedings of the Twenty First Annual ACM Symposium on Theory of Computing ACM Press, pp. 33-43

[23] Bertoni G, Daemen J, Peeters M and Van Assche G 2007 Sponge functions. In: Proceedings of the ECRYPT Hash Function Workshop

[24] Aumasson J P, Henzen L, Meier W and Phan R C W 2008 SHA-3 proposal BLAKE. Submission to the SHA-3 Competition

[25] Aumasson J P, Neves S, Wilcox-OHearn Z and Winnerlein C 2013 BLAKE2: simpler, smaller, fast as MD5. In: Proceedings of the International Conference on Applied Cryptography and Network Security, pp. 119-135

[26] Sobti R and Geetha G 2015 Performance comparison of Keccak, Skein, Grstl, Blake and JH: SHA-3 final round candidate algorithms on ARM Cortex A8 Processor. Int. J. Security Appl. 9(12): pp. 367-384, doi:10.14257/ijsia. 2015 
[27] Cremers C J F 2006 Scyther-semantics and verification of security protocols. PhD Thesis, Eindhoven University of Technology

[28] Chang D, Lee S, Nandi M and Yung M 2006 Indifferentiable security analysis of popular hash functions with prefix-free padding. In: ASIACRYPT, Lecture Notes in Computer Science, vol. 4284, pp. 283-298

[29] Biham E and Dunkelman O 2007 HAIFA: a framework for iterative hash functions. Cryptology ePrint Archive, Report 2007/278

[30] Bertoni G, Daemen J, Peeters M and Van Assche G 2008 On the indifferentiability of the sponge construction. In: $A d$ vances in Cryptology - EUROCRYPT 2008, Lecture Notes in Computer Science, vol. 4965, pp. 181-197

[31] Lavanya M and Natarajan V 2015 Impementation of ECDSA using sponge based hash function. In: Computational Intelligence, Cyber Security and Computational Models, Advances in Intelligent Systems and Computing, vol. 412, pp. 349-359

[32] Grover K and Lim A 2015 A survey of broadcast authentication schemes for wireless networks. Ad Hoc Netw. 24: 288-316

[33] Ma L, Ge Y and Zhu Y 2014 TinyZKP: a lightweight authentication scheme based on zero-knowledge proof for wireless body area networks. Wireless Pers. Commun. 77(2): 1077-1090

[34] Chowdhury A R, Chatterjee T and DasBit S 2014 LOCHA: a light-weight one-way cryptographic hash algorithm for wireless sensor network. Procedia Comput. Sci. 32: 497-504
[35] Lo C -M, Hwang T and Li C -M 2007 Revocation-free public-key encryption based on security-mediated public-key infrastructure. IET Inf. Security 3: 134-141

[36] Ge M, Choo K K R, Wu H and Yu Y 2016 Survey on key revocation mechanisms in wireless sensor networks. J. Netw. Comput. Appl. 63: 24-38

[37] Dong Z, Espejo R, Wan Y and Zhuang W 2015 Detecting and locating man-in-the-middle attacks in fixed wireless networks. J. Comput. Inf. Technol. 4: 283-293, doi:10.2498/ cit. 1002530

[38] Zargar S T, Joshi J and Tipper D 2013 A survey of defense mechanisms against distributed denial of service (DDoS) flooding attacks. IEEE Commun. Surveys Tuts. 15(4): 2046-2069

[39] Kitten T 2013 DDoS: lessons from phase 2 attacks, http:// www.bankinfosecurity.com/ddos-attacks-lessons-fromphase-2-a-5420/op-1

[40] Johnson D, Menezes A and Vanstone S 2001 The elliptic curve digital signature algorithm (ECDSA). Canada: Certicom Research

[41] Vanstone S 2004 Crypto column: the importance of good crypto and security standards. Code Cipher - Certicom Bull. Security Cryptogr. 1(4): www.certicom.com/codeandcipher

[42] Rondepierre F 2013 Revisiting atomic patterns for scalar multiplications on elliptic curves. In: Proceedings of CARDIS 2013 